\title{
Safety Assessment of Lactobacillus salivarius REN, a Probiotic Strain Isolated from
}

\section{Centenarian Feces}

\author{
Hao ZHANG ${ }^{1,2}$, Jing SuN ${ }^{1}$, Qing-yu $\mathrm{WANG}^{1}$, Yu-tong HE ${ }^{1}$, Hui-yong Gu${ }^{1}$, Hui-yuan Guo ${ }^{1}$, Qing-bo Ding ${ }^{3}$, \\ Jun-si YANG $^{1}$ and Fa-zheng REN ${ }^{1,2^{*}}$ \\ ${ }^{I}$ Beijing Laboratory for Food Quality and Safety, and Key Laboratory of Functional Dairy, College of Food Science and Nutritional \\ Engineering, China Agricultural University, Beijing 100083, China \\ ${ }^{2}$ Beijing Higher Institution Engineering Research Center of Animal Product, Beijing 100083, China \\ ${ }^{3}$ COFCO Limited, Beijing 10020, China
}

Received May 31, 2013; Accepted July 22, 2013

The aim of this study was to evaluate the oral safety of Lactobacillus salivarius REN, a probiotic isolated from the fecal samples of a healthy centenarian. BALB/c mice were respectively orally administered with $0,3 \times 10^{8}, 5 \times 10^{9}$, or $2 \times 10^{10} \mathrm{CFU} /$ mouse daily for 28 days, or injected intraperitoneally with $5 \times 10^{9}$ CFU/mouse in a single dose, then killed at day 2 and day 5 after injection, respectively. The results indicated that oral administration of Lactobacillus salivarius REN had no adverse effects on body weight, food intake, hematological and serum biochemical parameters, and there was no treatment-associated bacterial translocation to the blood, liver, spleen, or kidney. Intraperitoneal injection caused bacterial translocation to the liver, spleen, and kidney, except blood, the translocation did not trigger illness or death. This study suggests Lactobacillus salivarius REN is likely to be safe for human consumption.

Keywords: Lactobacillus salivarius REN, oral toxicity, safety, translocation

\section{Introduction}

Probiotics have received increasing attention over recent years, because of their human health benefits. Probiotics have anti-infection properties (Isolauri et al., 1991), and they can help inflammatory bowel disease (Bai and Ouyang, 2006; Ewaschuk and Dieleman, 2006; Mach, 2006) and irritable bowel syndrome (Drouault-Holowacz et al., 2008; McFarland and Dublin, 2008). However, the safety of probiotic bacteria has also raised concern due to some causative pathogenic cases. Some probiotics are reported to be associated with clinical diseases such as sepsis (Salminen et al., 2004), endocarditis (Aguirre and Collins, 1993), liver abscesses (Cukovic-Cavka et al., 2006), and urinary tract infections (Aznar et al., 2004). Probiotics are widely used in food throughout the world because of their beneficial effects, thus safety issues and side effects should be considered before their application (Abe et al., 2010).

Lactobacillus salivarius has received attention in recent

*To whom correspondence should be addressed. E-mail: renfazheng@263.net years as a promising probiotic species and it is increasingly employed in probiotic agents for animals and humans (Raftis et al., 2011). L. salivarius was isolated from the mammalian digestive tract as a candidate probiotic. The immunomodulatory properties of $L$. salivarius were confirmed by the alleviation of intestinal disease and the promotion of host wellbeing in animals and humans. The ability of L. salivarius to inhibit pathogens and tolerate host antimicrobial defenses demonstrates the adaptation of this species to the gastrointestinal niche (Neville and O'Toole, 2010).

A new probiotic strain, Lactobacillus salivarius REN (CGMCC 3606), was recently isolated by our laboratory from the fecal sample of a healthy centenarian living in Bama, Guangxi Zhuang Autonomous Region, China (Zhang et al., 2011). This strain exhibited antigenotoxic activity against 4-nitroquinoline-N-oxide (Wang et al., 2008) and antimicrobial activity against a Helicobacter strain that was initially isolated from carcinogen-treated rat intestinal microflora (Zhang et al., 2011). Due to the uncertainty of this strain's safety, a safety assessment was required before industrial application. In this study, a 28-day repeated oral dose 
study was conducted in healthy BALB/c mice, the animal health, hematological and serum biochemical parameters and the translocation ability of $L$. salivarius REN were investigated. Intraperitoneal injection with $L$. salivarius REN was also adopted in order to preclude possible toxicity caused by potential bacteria translocation. The harmful effects of translocation were also evaluated.

\section{Materials and Methods}

Bacterial strains The strain L. salivarius REN was isolated by our laboratory (Key Laboratory of Functional Dairy, China Agricultural University, Beijing, China). Stock cultures were maintained at $-80^{\circ} \mathrm{C}$ in $30 \%$ glycerol $(\mathrm{v} / \mathrm{v})$. The test strain was prepared daily during animal experiment. They were anaerobically cultured in the de Man Rogosa Sharpe (MRS; Aoboxing, Beijing, China) medium at $37^{\circ} \mathrm{C}$ by using 825-A anaerobic jars (Shenyang Fifth People Hospital, Shenyang, Liaoning, China) and AnaeroPack ${ }^{\circledR}$-Anaero (Mitsubishi Gas Chemical Company, Inc., Tokyo, Japan). Next, the strain cells were harvested by centrifugation, washed by distilled water twice, then resuspended in skim milk or saline solution.

Animals Seventy female BALB/c mice aged from 6 to 8 weeks were purchased from Vital River Lab Animal Technology Co. (Beijing, China). They were maintained under standard conditions (temperature $22 \pm 2^{\circ} \mathrm{C}$, humidity $55 \pm 2 \%$, $12 \mathrm{~h}$ light-dark cycle) throughout the study. Animals were fed ad libitum with normal diet (Vital River Lab Animal Technology Co., Beijing, China) and they had free access to water during the experimental period. The Animal maintenance and experimental procedures were approved by the Animal Ethics Committee of China Agricultural University.

Experimental design After 7 days of acclimation, 70 mice were randomly assigned into six experimental groups. Four groups ( $\mathrm{n}=10$ per group) were orally administered bacteria suspended in skim milk with 0 (control), $3 \times 10^{8}, 5 \times$ $10^{9}$, or $2 \times 10^{10} \mathrm{CFU} /$ mouse per day for 28 days, respectively. One group $(\mathrm{n}=20)$ was injected intraperitoneally with $5 \times$ $10^{9} \mathrm{CFU} /$ mouse suspended in saline solution at a single dose, and 10 mice of them were killed at day 2 and day 5 after injection, respectively (Lara-Villoslada et al., 2007a). The last group $(\mathrm{n}=10)$ only injected with saline solution and killed at day 0 as control. The activity, behavior, and general health of animals were observed daily throughout the experiment. Body weight (BW) and food intake were recorded once a week. At the end of the experiment, all mice were fasted for $12 \mathrm{~h}$ before anaesthetized by intraperitoneal administration of sodium pentothal $(50 \mathrm{mg} / \mathrm{kg} \mathrm{BW})$ (Lara-Villoslada et al., 2007b). Blood samples were collected into EDTA-containing tubes by cardiac puncture in sterile conditions, and serum was obtained by centrifugation $3000 \times \mathrm{g}$ for $20 \mathrm{~min}$ at $4^{\circ} \mathrm{C}$. Then the animals were sacrificed and the liver, spleen, and kidney were removed in sterile conditions and weighed.

Hematological and serum biochemical examination The hematological parameters were measured using an automated hematology analyzer (XS-1000i, Sysmex; Kobe, Japan), i.e., white blood cell count (WBC), red blood cell count (RBC), hemoglobin $(\mathrm{Hb})$, hematocrit $(\mathrm{Ht})$, mean cell volume (MCV), mean cell hemoglobin (MCH), mean cell hemoglobin concentration (MCHC), and plate count (PLT). Clinical biochemical parameters were measured using an automated biochemical analyzer (Hitachi 7080, Tokyo, Japan), i.e., alanine aminotransferase (ALT), asparatate aminotransferase (AST), total protein (TP), albumin (Alb), globulin (Gb), A/G ratio, total cholesterol (TC), glucose (Glu), blood urea nitrogen (BUN), creatinine $(\mathrm{Cr})$, and calcium $\left(\mathrm{Ca}^{2+}\right)$.

Bacterial translocation Bacterial translocation was analyzed in the blood, liver, spleen, and kidney. A $50 \mu \mathrm{L}$ sample of blood was cultured onto MRS agar medium and brain heart infusion (BHI) agar (Beijing Land Bridge Technology Co. Ltd., Beijing, China) and incubated at $37^{\circ} \mathrm{C}$ for $48 \mathrm{~h}$, anaerobically for MRS and aerobically for BHI. Tissue samples were homogenized in buffered peptone water $(1 \mathrm{~g} / \mathrm{mL})$ and $100 \mu \mathrm{L}$ of the homogenates were cultured onto MRS and $\mathrm{BHI}$ agar as previously mentioned. Colonies were enumerated after $48 \mathrm{~h}$ and the results were expressed as the incidence of translocation (the number of mice where translocation was detected/total number of mice). Positive growth on agar plates was defined by the presence of even a single colony of any microorganisms (Lara-Villoslada et al., 2009).

Identification of bacteria by PCR L. salivarius REN detection was performed by PCR with the DNA samples taken directly from MRS and BHI agar colonies. PCR used the primers 5'-AATCGCTAAACTCATAACCT- ${ }^{\prime}$ ' and 5'-CACTCTCTTTGGCTAATCTT-3' (GenBank accession No.: AF182725.1) reported by Drisko et al. (2005), and synthesized by Invitrogen Biotech Co. Ltd. (Beijing, China).

Statistical analysis Results are expressed as means \pm standard deviations (SD). Data on body weight, food consumption, organ weight, hematological and biochemical parameters were tested by Dunnett's method. Differences between bacterial translocation incidences were analyzed using the chi-square test. All statistical analyses of data were performed using SPSS 13.0 (SPSS, Inc., Chicago, IL, USA), with the statistical significance level at $P<0.05$.

\section{Results and Discussion}

Safety issues of Lactobacillus salivarius, including possible side effects, should be considered before application due to its uncertain safety (Abe et al., 2010). Oral safety as- 
sessment is commonly used in safety evaluation of probiotic (Duangjitcharoen et al., 2009; Perelmuter et al., 2011), thus we conducted a 28 -day repeated oral dose study. No noticeable activity or behavioral changes were observed in mice during the experimental protocol, and no illness or death occurred. Oral administration of different doses of L. salivarius REN caused no significant difference in BW, food intake, and tissue weight among the groups throughout the experiment (Table 1), which are similar with previous reports (Zhou et al., 2000; Lara-Villoslada et al., 2007a). These results demonstrate that $L$. salivarius REN does not exhibit gross oral toxicity on the animals' health status, growth and development, even in high dose groups.

In order to evaluate the adverse sub-clinical effects of $L$. salivarius REN, hematological and general blood biochemical parameters were monitored (Zhou et al., 2000). Hematological parameters are usually used to represent the deleterious effects of test substance on the blood (Lee et al., 2010), when the composition of blood cells change, it might indicate the pathological changes in bone marrow or the reaction of immune cells (Yakubu et al., 2007). Biochemical assays are used to detect mild nutrient deficiencies or imbalance in

Table 1. Body and organ weights of mice orally treated with different doses of L. salivarius REN for 28 days ${ }^{\mathrm{a}}$.

\begin{tabular}{lcccc}
\hline & \multicolumn{4}{c}{ Oral dose (CFU/mouse/d) } \\
\cline { 2 - 4 } & 0 (Control) & $3 \times 10^{8}$ & $5 \times 10^{9}$ & $2 \times 10^{10}$ \\
\hline Initial weight (g) & $18.47 \pm 1.16$ & $17.11 \pm 1.18$ & $18.24 \pm 1.25$ & $18.66 \pm 1.01$ \\
Final weight (g) & $20.85 \pm 0.84$ & $19.31 \pm 1.03$ & $20.84 \pm 1.25$ & $20.92 \pm 0.93$ \\
Weight gain (\%) & $13.02 \pm 2.80$ & $12.97 \pm 3.22$ & $14.31 \pm 1.92$ & $12.16 \pm 1.56$ \\
Food intake (g/mouse/d) & $3.07 \pm 0.45$ & $2.73 \pm 0.38$ & $3.01 \pm 0.19$ & $2.41 \pm 0.37$ \\
Liver weight (mg) & $1216 \pm 105$ & $1170 \pm 190$ & $1110 \pm 115$ & $1094 \pm 135$ \\
Spleen weight (mg) & $110 \pm 13$ & $113 \pm 25$ & $114 \pm 17$ & $118 \pm 18$ \\
Kidney weight (mg) & $320 \pm 25$ & $320 \pm 26$ & $315 \pm 29$ & $300 \pm 26$ \\
\hline
\end{tabular}

${ }^{\mathrm{a}}$ Data are presented as means $\pm \mathrm{SD}$.

Table 2. Hematological and serum biochemical parameters of mice orally treated with different doses of L. salivarius REN for 28 days ${ }^{\mathrm{a}}$.

\begin{tabular}{|c|c|c|c|c|}
\hline & \multicolumn{4}{|c|}{ Oral dose (CFU/mouse/d) } \\
\hline & 0 (Control) & $3 \times 10^{8}$ & $5 \times 10^{9}$ & $2 \times 10^{10}$ \\
\hline \multicolumn{5}{|c|}{ Hematological parameters } \\
\hline $\operatorname{WBC}\left(10^{9} / \mathrm{L}\right)$ & $6.5 \pm 0.8$ & $6.8 \pm 1.1$ & $7.2 \pm 1.5$ & $6.9 \pm 1.3$ \\
\hline $\operatorname{RBC}\left(10^{12} / \mathrm{L}\right)$ & $9.2 \pm 0.4$ & $8.8 \pm 0.4$ & $9.1 \pm 0.4$ & $8.7 \pm 0.3$ \\
\hline $\mathrm{Hb}(\mathrm{g} / \mathrm{L})$ & $157.9 \pm 6.7$ & $164.3 \pm 4.5$ & $156.2 \pm 4.5$ & $158.3 \pm 4.2$ \\
\hline $\mathrm{Ht}(\%)$ & $47.8 \pm 2.6$ & $45.3 \pm 2.2$ & $47.8 \pm 2.3$ & $45.2 \pm 1.4$ \\
\hline $\operatorname{MCV}(\mathrm{fl})$ & $52.1 \pm 0.7$ & $52.0 \pm 0.9$ & $52.3 \pm 0.6$ & $51.6 \pm 0.9$ \\
\hline $\mathrm{MCH}(\mathrm{pg})$ & $17.2 \pm 0.4$ & $18.8 \pm 0.8$ & $17.2 \pm 0.4$ & $18.3 \pm 0.6$ \\
\hline $\mathrm{MCHC}(\mathrm{g} / \mathrm{L})$ & $330.6 \pm 11.4$ & $360.4 \pm 10.8$ & $330.7 \pm 8.8$ & $350.9 \pm 14.7$ \\
\hline $\operatorname{PLT}\left(10^{9} / \mathrm{L}\right)$ & $1316.8 \pm 250.4$ & $1193.9 \pm 111.0$ & $1135.1 \pm 117.8$ & $1136.6 \pm 66.5$ \\
\hline \multicolumn{5}{|c|}{ Serum biochemical parameters } \\
\hline ALT (U/L) & $39.38 \pm 3.11$ & $38.70 \pm 4.62$ & $38.80 \pm 3.71$ & $36.78 \pm 2.77$ \\
\hline AST (U/L) & $104.60 \pm 11.84$ & $109.00 \pm 15.73$ & $96.90 \pm 11.45$ & $105.90 \pm 25.54$ \\
\hline $\mathrm{TP}(\mathrm{g} / \mathrm{L})$ & $53.70 \pm 2.41$ & $53.30 \pm 1.83$ & $53.00 \pm 1.33$ & $54.00 \pm 1.70$ \\
\hline $\mathrm{Alb}(\mathrm{g} / \mathrm{L})$ & $36.20 \pm 0.92$ & $36.20 \pm 1.14$ & $35.70 \pm 0.48$ & $36.30 \pm 1.16$ \\
\hline $\mathrm{Gb}(\mathrm{g} / \mathrm{L})$ & $17.89 \pm 1.27$ & $17.10 \pm 1.10$ & $17.30 \pm 1.25$ & $17.70 \pm 0.82$ \\
\hline $\mathrm{A} / \mathrm{G}$ ratio & $2.04 \pm 0.13$ & $2.12 \pm 0.13$ & $2.07 \pm 0.15$ & $2.05 \pm 0.09$ \\
\hline $\mathrm{TC}(\mathrm{mmol} / \mathrm{L})$ & $2.43 \pm 0.23$ & $2.45 \pm 0.13$ & $2.54 \pm 0.33$ & $2.50 \pm 0.18$ \\
\hline Glu (mmol/L) & $2.96 \pm 0.86$ & $2.65 \pm 0.35$ & $3.34 \pm 0.70$ & $3.31 \pm 0.79$ \\
\hline BUN (mmol/L) & $13.29 \pm 3.47$ & $13.41 \pm 3.40$ & $13.11 \pm 2.10$ & $14.19 \pm 2.00$ \\
\hline $\mathrm{Cr}(\mu \mathrm{mol} / \mathrm{L})$ & $11.11 \pm 2.36$ & $11.26 \pm 2.32$ & $11.76 \pm 2.76$ & $13.86 \pm 3.47$ \\
\hline $\mathrm{Ca}^{2+}(\mathrm{mmol} / \mathrm{L})$ & $2.31 \pm 0.05$ & $2.32 \pm 0.04$ & $2.24 \pm 0.16$ & $2.30 \pm 0.07$ \\
\hline
\end{tabular}

${ }^{\mathrm{a}}$ Data are presented as means $\pm \mathrm{SD}$. WBC, white blood cell count; RBC, red blood cell count; Hb, hemoglobin, Ht, hematocrit; MCV, mean cell volume; $\mathrm{MCH}$, mean cell hemoglobin; $\mathrm{MCHC}$, mean cell hemoglobin concentration; PLT, plate count; ALT, alanine aminotransferase; AST, asparatate aminotransferase; TP, total protein; Alb, albumin; Gb, globulin; A/G ratio, albumin/globulin ratio; TC, total cholesterol; Glu, glucose; BUN, blood urea nitrogen; $\mathrm{Cr}$, creatinine; $\mathrm{Ca}^{2+}$, calcium. 
nutrient metabolism (Swendseid, 1987), which usually apparent before any clinical symptoms (Zhou et al., 2000). The changes of serum biochemical parameters might indicate responsive organs were affected or even damaged (Swendseid, 1987). The current study showed that oral administration of L. salivarius REN for 28 days had no adverse effects on the hematology and blood biochemistry (Table 2). Therefore, our results suggest that oral administration of L. salivarius REN did not cause the pathological and metabolic changes.

Bacteria translocation or bacteremia indicates the degree of bacterial infectivity and pathogenicity (Urao et al., 1996). Bacterial translocation is regarded as a potential indicator of probiotic toxicity, because it is the first step in the pathogenesis process for many opportunistic indigenous lumen strains (Steffen and Berg, 1983). As shown in the Table 3, there were no significant differences in the incidence of translocation to the liver, spleen, or kidney between the control and treated groups at any of tested doses. Although the translocation happened after oral administration of L. salivarius REN, the colonies detected on the agar plates did not match $L$. salivarius REN (data not shown) by conventional PCR using specific primer, suggesting that translocation was not associated with L. salivarius REN treatment. Instead, this translocation may be caused by some indigenous bacteria, since the presence of bacteria in the organs was reported in other research (Lara-Villoslada et al., 2007a; Lara-Villoslada et al., 2007b). The present study detected no bacteremia cases in any of the oral-treated groups, despite the high doses of bacteria administered to mice. These data show that oral administration of high doses of L. salivarius REN did not cause bacterial translocation to the blood, spleen, kidney, or liver of mice.

To further evaluate any possible adverse effects, translocation was induced by intraperitoneal injection with $L$. salivarius REN (Lara-Villoslada et al., 2007a). No behavioral changes or death was observed in the injected mice after bacterial translocation. The injection had no significant effect on the BW and food intake, but the liver, spleen, and kidney weight were significantly higher at day 5 compared with the control (Table 4). Intraperitoneal administration also led to a lower PLT level at day 2 and a higher PLT level at day 5 after injection (Table 5), and that was probably caused

Table 3. Incidence of bacterial translocation of mice orally treated with different doses of L. salivarius REN for 28 days ${ }^{\mathrm{a}}$.

\begin{tabular}{ccccc}
\hline & \multicolumn{3}{c}{ Oral dose $(\mathrm{CFU} / \mathrm{mouse} / \mathrm{d})$} \\
\cline { 2 - 5 } & 0 (Control) & $3 \times 10^{8}$ & $5 \times 10^{9}$ & $2 \times 10^{10}$ \\
\hline Liver & & & & \\
MRS & $1 / 10(25)$ & $0 / 10$ & $0 / 10$ & $0 / 10$ \\
BHI & $0 / 10$ & $0 / 10$ & $0 / 10$ & $0 / 10$ \\
Spleen & & & & $0 / 10$ \\
MRS & $2 / 10(35-122)$ & $0 / 10$ & $2 / 10(4-26)$ & $0 / 10$ \\
BHI & $1 / 10(56)$ & $0 / 10$ & $0 / 10$ & $0 / 10$ \\
Kidney & & & & $0 / 10$ \\
MRS & $2 / 10(5-184)$ & $0 / 10$ & $1 / 10(220)$ & $0 / 10$ \\
BHI & $0 / 10$ & $0 / 10$ & $0 / 10$ & $0 / 10$ \\
Blood & & & & $0 / 10$ \\
MRS & $0 / 10$ & $0 / 10$ & $0 / 10$ & \\
BHI & $0 / 10$ & $0 / 10$ & & \\
\hline
\end{tabular}

${ }^{a}$ Numbers in parentheses represent the range of bacteria which expressed as CFU per organ.

Table 4. Body and organ weights of mice intraperitoneally injected with $5 \times 10^{9} \mathrm{CFU} /$ mouse L. salivarius REN at day 2 and day 5 after the injection ${ }^{\mathrm{a}}$.

\begin{tabular}{lccc}
\hline & Control & Day 2 & Day 5 \\
\hline Initial weight $(\mathrm{g})$ & $18.33 \pm 0.74$ & $17.71 \pm 1.47$ & $18.05 \pm 0.87$ \\
Final weight $(\mathrm{g})$ & $18.74 \pm 0.49$ & $18.16 \pm 1.60$ & $18.84 \pm 1.08$ \\
Food intake $(\mathrm{g} / \mathrm{mouse} / \mathrm{d})$ & $2.71 \pm 0.23$ & $2.07 \pm 0.35$ & $2.54 \pm 0.37$ \\
Liver weight $(\mathrm{mg})$ & $887 \pm 116$ & $909 \pm 63$ & $1026 \pm 11^{*}$ \\
Spleen weight $(\mathrm{mg})$ & $89 \pm 10$ & $92 \pm 17$ & $104 \pm 10^{*}$ \\
Kidney weight $(\mathrm{mg})$ & $230 \pm 15$ & $238 \pm 24$ & $267 \pm 14^{*}$ \\
\hline
\end{tabular}

${ }^{a}$ Data are presented as means $\pm \mathrm{SD} .{ }^{*} P<0.05$ vs. control. 
Table 5. Hematological and serum biochemical parameters of mice intraperitoneally injected with $5 \times 10^{9}$ CFU/mouse L. salivarius REN at day 2 and day 5 after the injection ${ }^{\mathrm{a}}$.

\begin{tabular}{lccc}
\hline & Control & Day 2 & Day 5 \\
\hline Hematological parameters & & & \\
WBC $\left(10^{9} / \mathrm{L}\right)$ & $7.1 \pm 0.9$ & $7.2 \pm 0.5$ & $7.3 \pm 0.9$ \\
$\mathrm{RBC}\left(10^{12} / \mathrm{L}\right)$ & $8.1 \pm 0.4$ & $8.4 \pm 0.4$ & $8.0 \pm 0.3$ \\
$\mathrm{Hb}(\mathrm{g} / \mathrm{L})$ & $144.8 \pm 5.3$ & $146.6 \pm 3.7$ & $144.0 \pm 5.9$ \\
$\mathrm{Ht}(\%)$ & $43.3 \pm 1.7$ & $44.4 \pm 2.7$ & $43.9 \pm 2.2$ \\
$\mathrm{MCV}(\mathrm{fL})$ & $53.3 \pm 1.2$ & $52.6 \pm 0.9$ & $53.8 \pm 0.6$ \\
$\mathrm{MCH}(\mathrm{pg})$ & $17.8 \pm 0.5$ & $17.8 \pm 1.3$ & $18.2 \pm 0.3$ \\
$\mathrm{MCHC}(\mathrm{g} / \mathrm{L})$ & $334.5 \pm 6.5$ & $343.7 \pm 20.2$ & $337.2 \pm 4.6$ \\
$\mathrm{PLT}\left(10^{9} / \mathrm{L}\right)$ & $1093.1 \pm 182.2$ & $941 \pm 139.7 *$ & $1670.9 \pm 129.3 *$ \\
$\mathrm{Serum}$ biochemical parameters & & & \\
$\mathrm{ALT}(\mathrm{U} / \mathrm{L})$ & $36.92 \pm 4.44$ & $33.10 \pm 4.12$ & $31.08 \pm 4.89^{*}$ \\
$\mathrm{AST}(\mathrm{U} / \mathrm{L})$ & $104.10 \pm 19.73$ & $82.10 \pm 14.74^{*}$ & $98.56 \pm 9.94$ \\
$\mathrm{TP}(\mathrm{g} / \mathrm{L})$ & $55.90 \pm 6.74$ & $50.10 \pm 2.28^{*}$ & $53.12 \pm 1.72^{*}$ \\
$\mathrm{Alb}(\mathrm{g} / \mathrm{L})$ & $36.60 \pm 3.31$ & $32.10 \pm 0.99 *$ & $34.17 \pm 1.05^{*}$ \\
$\mathrm{~Gb}(\mathrm{~g} / \mathrm{L})$ & $19.30 \pm 3.47$ & $18.00 \pm 1.56$ & $19.12 \pm 1.12$ \\
$\mathrm{~A} / \mathrm{G} \mathrm{ratio}$ & $1.90 \pm 0.18$ & $1.79 \pm 0.13$ & $1.80 \pm 0.17$ \\
$\mathrm{TC}(\mathrm{mmol} / \mathrm{L})$ & $2.80 \pm 0.47$ & $2.78 \pm 0.27$ & $2.85 \pm 0.24$ \\
$\mathrm{Glu}(\mathrm{mmol} / \mathrm{L})$ & $2.47 \pm 0.22$ & $2.58 \pm 0.31$ & $2.40 \pm 0.15$ \\
$\mathrm{BUN}(\mathrm{mmol} / \mathrm{L})$ & $12.98 \pm 0.93$ & $13.58 \pm 1.69$ & $11.38 \pm 1.46$ \\
$\mathrm{Cr}(\mu \mathrm{mol} / \mathrm{L})$ & $11.99 \pm 1.40$ & $12.27 \pm 1.69$ & $11.74 \pm 0.82$ \\
$\mathrm{Ca}{ }^{2+}(\mathrm{mmol} / \mathrm{L})$ & $2.23 \pm 0.07$ & $2.18 \pm 0.06$ & $2.28 \pm 0.07$ \\
\hline
\end{tabular}

${ }^{\mathrm{a}}$ Data are presented as means $\pm \mathrm{SD} . * P<0.05$ vs. control. WBC, white blood cell count; $\mathrm{RBC}$, red blood cell count; $\mathrm{Hb}$, hemoglobin, $\mathrm{Ht}$, hematocrit; $\mathrm{MCV}$, mean cell volume; $\mathrm{MCH}$, mean cell hemoglobin; $\mathrm{MCHC}$, mean cell hemoglobin concentration; PLT, plate count; ALT, alanine aminotransferase; AST, asparatate aminotransferase; TP, total protein; Alb, albumin; Gb, globulin; A/G ratio, albumin/globulin ratio; TC, total cholesterol; Glu, glucose; BUN, blood urea nitrogen; $\mathrm{Cr}$, creatinine; $\mathrm{Ca}^{2+}$, calcium.

Table 6. Incidence of bacterial translocation of mice intraperitoneally injected with $5 \times 10^{9}$ CFU/mouse L. salivarius REN at day 2 and day 5 after the injection ${ }^{\mathrm{a}}$.

\begin{tabular}{cccc}
\hline & Control & Day 2 & Day 5 \\
\hline Liver & & & \\
MRS & $1 / 10(8)$ & $10 / 10(536-1680)^{*}$ & $4 / 10(4-29)$ \\
BHI & $0 / 10$ & $10 / 10(270-1920)^{*}$ & $2 / 10(8-12)$ \\
Spleen & & & \\
MRS & $1 / 10(78)$ & $10 / 10(130-2040)^{*}$ & $5 / 10(5-27)$ \\
BHI & $2 / 10(50-223)$ & $10 / 10(108-1760)^{*}$ & $2 / 10(4-20)$ \\
Kidney & & & \\
MRS & $1 / 10(43)$ & $10 / 10(77-1312)^{*}$ & $0 / 10$ \\
BHI & $0 / 10$ & $10 / 10(73-1600)^{*}$ & $0 / 10$ \\
Blood & $0 / 10$ & & $0 / 10$ \\
MRS & $0 / 10$ & $0 / 10$ & $0 / 10$ \\
BHI & & $0 / 10$ & \\
\hline
\end{tabular}

${ }^{\text {a }}$ Numbers in parentheses represent the range of bacteria which expressed as CFU per organ. $* P<$ 0.05 vs. control.

by a transient inflammatory process which could be maintained over time (Lara-Villoslada et al., 2007a). As for serum biochemical parameters, the results suggested there was significantly lower $(P<0.05)$ for ALT 5 day after injection when compared to control. AST was significantly lower 2 day after injection, although it was similar to that of control mice at 5 day. TP and Alb were significantly lower in both 2 day and 5 day after injection than the control (Table 5). Considering that no signs of illness were observed in our study, more studies are needed to clarify the influence. As shown in Table 6, there was a significant translocation of $L$. salivarius REN to the liver, spleen and kidney 2 day after intraperito- 
neal injection. However, the incidence of translocation was lower at day 5 and the strain was not observed in the kidney. Bacteremia was not observed in any of the experimental groups.

\section{Conclusions}

In the present study, for the first time, a safety assessment of the probiotic L. salivarius REN was performed by oral administration and intraperitoneal injection. Mice fed with $L$. salivarius $\mathrm{REN}$ at high dose for 28 days exhibited no deleterious effects on their body weight, hematological, or blood biochemical parameters. There was no bacteria translocation to the blood, liver, spleen or kidney in mice after 28day oral administration. In conclusion, our results suggested Lactobacillus salivarius REN is likely to be safe for human consumption.

Acknowledgements We gratefully acknowledged the financial support received by National Science and Technology Support Program (2012BAD28B07 and 2011AA100903).

\section{References}

Abe, F., Muto, M., Yaeshima, T., Iwatsuki, K., Aihara, H., Ohashi, Y. and Fujisawa, T. (2010). Safety evaluation of probiotic bifidobacteria by analysis of mucin degradation activity and translocation ability. Anaerobe, 16, 131-136.

Aguirre, M. and Collins, M.D. (1993). Lactic acid bacteria and human clinical infection. J. Appl. Bacteriol., 75, 95-107.

Aznar, E., Buendía, B., Garcia-Penuela, E., Escudero, E., Alarcon, T. and Lopez-Brea, M. (2004). Community-acquired urinary tract infection caused by vancomycin-resistant Enterococcus faecalis clinical isolate. Rev. Esp. Quimioter, 17, 263-265.

Bai, A.P. and Ouyang, Q. (2006). Probiotics and inflammatory bowel diseases. Postgrad. Med. J., 82, 376-382.

Cukovic-Cavka, S., Likic, R., Francetic, I., Rustemovic, N., Opacic, M. and Vucelic, B. (2006). Lactobacillus acidophilus as a cause of liver abscess in a NOD2/CARD15-positive patient with Crohn's disease. Digestion, 73, 107-110.

Drisko, J., Bischoff, B., Giles, C., Adelson, M., Rao, RVS. and McCallum, R. (2005). Evaluation of five probiotic products for label claims by DNA extraction and polymerase chain reaction analysis. Dig. Dis. Sci., 50, 1113-1117.

Drouault-Holowacz, S., Bieuvelet, S., Burckel, A., Cazaubiel, M., Dray, X. and Marteau, P. (2008). A double blind randomized controlled trial of a probiotic combination in 100 patients with irritable bowel syndrome. Gastroenterol. Clin. Biol., 32, 147-152.

Duangjitcharoen, Y., Kantachote, D., Ongsakul, M., Poosaran, N. and Chaiyasut, C. (2009). Potential use of probiotic Lactobacillus plantarum SS2 isolated from a fermented plant beverage: safety assessment and persistence in the murine gastrointestinal tract.
World J. Microbiol. Biotechnol., 25, 315-321.

Ewaschuk, J.B. and Dieleman, L.A. (2006). Probiotics and prebiotics in chronic inflammatory bowel diseases. World J. Gastroenterol., 12, 5941-5950.

Isolauri, E., Juntunen, M., Rautanen, T., Sillanaukee, P. and Koivula, T. (1991). A human lactobacillus strain (Lactobacillus casei $s p$ strain $G G$ ) promotes recovery from acute diarrhea in children. Pediatrics, 88, 90-97.

Lara-Villoslada, F., Sierra, S., Diaz-Ropero, M.P., Olivares, M. and Xaus, J. (2007a). Safety assessment of the human milk-isolated probiotic Lactobacillus salivarius CECT5713. J. Dairy Sci., 90, 3583-3589.

Lara-Villoslada, F., Sierra, S., Diaz-Ropero, M.P., Rodriguez, J.M., Xaus, J. and Olivares, M. (2009). Safety Assessment of Lactobacillus fermentum CECT5716, a probiotic strain isolated from human milk. J. Dairy Res., 76, 216-221.

Lara-Villoslada, F., Sierra, S., Martin, R., Delgado, S., Rodriguez, J.M., Olivares, M. and Xaus, J. (2007b). Safety assessment of two probiotic strains, Lactobacillus coryniformis CECT5711 and Lactobacillus gasseri CECT5714. J. Appl. Microbiol., 103, 175184.

Lee, K.C., Liu, C.F., Lin, T.H. and Pan, T.M. (2010). Safety and risk assessment of the genetically modified Lactococci on rats intestinal bacterial flora. Int. J. Food Microbiol., 142, 164-169.

Mach, T. (2006). Clinical usefulness of probiotics in inflammatory bowel diseases. J. Physiol. Pharmacol., 57, 23-33.

McFarland, L.V. and Dublin, S. (2008). Meta-analysis of probiotics for the treatment of irritable bowel syndrome. World J. Gastroenterol., 14, 2650-2661.

Neville, B.A. and O'Toole, P.W. (2010). Probiotic properties of Lactobacillus salivarius and closely related Lactobacillus species. Future Microbiol., 5, 759-774.

Perelmuter, K., Fraga, M., Delucchi, L. and Zunino, P. (2011). Safety assessment and enteric colonization ability of a native canine Lactobacillus murinus strain. World J. Microbiol. Biotechnol., 27, 1725-1730.

Raftis, E.J., Salvetti, E., Torriani, S., Felis, G.E. and O'Toole, P.W. (2011). Genomic Diversity of Lactobacillus salivarius. Appl. Environ. Microbiol., 77, 954-965.

Salminen, M.K., Rautelin, H., Tynkkynen, S., Poussa, T., Saxelin, M., Valtonen, V. and Jarvinen, A. (2004). Lactobacillus bacteremia, clinical significance, and patient outcome, with special focus on Probiotic L. Rhamnosus GG. Clin. Infect. Dis., 38, 62-69.

Steffen, E.K. and Berg, R.D. (1983). Relationship between cecal population-levels of indigenous bacteria and translocation to the mesenteric lymph-nodes. Infect. Immun., 39, 1252-1259.

Swendseid, M.E. (1987). Biochemical assessment of protein and amino acid status. In "Nutritional Status Assessment of the Individual: Proceedings of a National Conference Sponsored by the Food and Nutrition Council of the American Health Foundation," 
ed. by G. E. Livingston. New York, New York, pp. 205-211.

Urao, M., Teitelbaum, D.H., Drongowski, R.A. and Coran, A.G. (1996). The association of gut-associated lymphoid tissue and bacterial translocation in the newborn rabbit. J. Pediatr. Surg., 31, $1482-1487$.

Wang, F., Jiang, L., Liu, A.P., Guo, X.H. and Ren, F.Z. (2008). Analysis of antigenotoxicity of Lactobacillus salivarius by high performance liquid chromatography. Fenxi Huaxue, 36, 740-744.

Yakubu, M., Akanji, M. and Oladiji, A. (2007). Hematological evaluation in male albino rats following chronic administration of aqueous extract of Fadogia agrestis stem. Pharmacogn. Mag., 3,
34.

Zhang, M., Qiao, X.W., Zhao, L., Jiang, L. and Ren, F.Z. (2011). Lactobacillus salivarius REN counteracted unfavorable 4-nitroquinoline-1-oxide-induced changes in colonic microflora of rats. J. Microbiol., 49, 877-883.

Zhou, J.S., Shu, Q., Rutherfurd, K.J., Prasad, J., Birtles, M.J., Gopal, P.K. and Gill, H.S. (2000). Safety assessment of potential probiotic lactic acid bacterial strains Lactobacillus rhamnosus HN001, Lb. acidophilus HN017, and Bifidobacterium lactis HN019 in BALB/c mice. Int. J. Food Microbiol., 56, 87-96. 